that approach, users mail in their samples, beamline staff generate the data, and the users analyse it remotely in real time, communicating with the synchrotron staff about whether or not more reads are necessary or if any adjustments need to be made.

Rosenbaum says institutional or cultural obstacles now make it difficult to attract and retain synchrotron staffers. Beamline operators, he says, are "consumed" by their users, and seldom work on their own projects. As a result, many find it hard to get first authorship on papers, making it difficult for them to earn the credentials necessary for a tenure-track position later in their careers. Without a stable staff, beamlines do not run as efficiently as they should, he says. Rosenbaum says the problem applies to synchrotrons throughout the world and extends beyond structural biology.

Sol Gruner, director of the Cornell High Energy Synchrotron Source (CHESS), says that his facility also has several openings.
Gruner notes that staff support at the facilities is more important than having the latest technology.

Although Gruner recruits computational specialists, structural biologists and physicists, he says the physicists - the people responsible for generating the light and seeing that it hits the sample - are the hardest to find.

That difficulty could reflect a general shortage of physicists in the United States. There are fewer physicists looking for work then biologists or chemists (see Nature 409, 445 ; 2001). The shortage may be especially pronounced at synchrotrons because qualified people must possess multidisciplinary skills. "You're talking about a small group of people who are very much in demand for their versatile talents," says Gruner.

Paul Smaglik is Naturejobs editor.

http://biosync.sdsc.edu

http://www.nigms.nih.gov/funding/psi.html

http://www.x12c.nsIs.bnl.gov/StrGen.htm

\title{
Staffing shortage threatens Japan's structural genomics
}

$\mathrm{S}$ ynchrotron radiation facilities in Japan are suffering from a chronic staff shortage. Despite considerable funding increases in some application areas such as structural genomics and nanotechnology — demand for beamline specialists, experimentalists, technicians and specialized software engineers is outstripping supply.

The bold move a few years ago to put structural genomics at the top of the agenda of the newly established RIKEN Genomic Sciences Center in Yokohama caused a stir within Japan's genetics research community. Although critics of the new centre pointed out that more emphasis should be put on genome sequencing, the centre's designated director, Akiyoshi Wada, argued that focusing efforts on structural genomics would constitute a more viable strategy for a "latecomer" in the genomics field.

With SPring-8, the world's largest synchrotron radiation facility, opening its doors for research in October 1997 in Harima, the timing for a structural genomics initiative was well chosen. A third-generation synchrotron radiation facility in the hard X-ray range, SPring-8 was planned and constructed jointly by RIKEN and the Japan Atomic Energy Research Institute (JAERI).

RIKEN presently occupies two of the four beamlines dedicated to structural biology at SPring-8. Using 'multiwavelength anomalous diffraction' and a device called a trichromator, Japanese scientists have successfully tackled the structural basis of glutamate receptors in the central nervous system, and the structure of various proteins includ- ing bovine rhodopsin.

Funding for structural genomics has been exploding in Japan over the past two years. But, according to insiders, virtually all synchrotron radiation facilities remain inadequately staffed and user support is limited. According to Masashi Miyano, who directs the structural biology group at RIKEN's Harima research centre, the number of beamline staff at SPring- 8 is about one-third that of the staff at comparable facilities outside Japan.

Automation may eventually provide part of the answer - especially for ambitious projects in structural genomics. One initiative, headed by Seiki Kuramitsu, a professor in the graduate school of science at Osaka University, aims to elucidate the structure and function of all proteins in the extreme thermophile Thermus thermophilius. For this project to succeed, automating the whole process, from protein production to crystallization and data acquisition, will become crucial because of the large number of proteins.

Beyond increasing automation and hiring more people, Japanese synchrotrons could be improved by focusing more on service, several users say. There is widespread agreement that both existing and future synchrotron research facilities in Japan must spend more time reflecting the needs of their users and prioritizing beam-time allocation. Some facilities within Japan are well known for their lack of user support. And users from the biology community are increasingly demanding standardization of the equipment and ease of use.

Physicists such as Tsuneaki Miyahara, a professor in the department of physics at the Tokyo Metropolitan University, disagree, arguing that it is important to teach scientists how to improve instrumentation, rather than just how to use it. "Younger scientists generally do not want to go into the details of instrumentation, which might crucially impact on the future development of synchrotron radiation research in Japan," says Miyahara.

But it is not just hardware specialists that are needed. Structural biologists spend most of their time on model development and verification, says Miyano. The software tools that are needed for this purpose are coming mostly from the United States. "Without a long-term strategy to develop adequate software capacities, the situation seems critical," says Miyano.

Robert Triendl is a freelance writer based in Tokyo.

Web links

SPring-8 http://www.spring8.or.jp

RIKEN Structural Genomics Initiative

http://www.rsgi.riken.go.jp

KSR http://wwwal.kuicr.kyoto-u.ac.jp KEK Photon Factory http://www.kek.jp

UVSOR http://vsor-ntserver.ims.ac.jp

HiSOR http://www.hsrc.hiroshima-u.ac.jp

Ritsumeikan University SR Center

http://www.ritsumei.ac.jp/se/d11/index-e.html New Subaru

http://www.lasti.himeji-tech.ac.jp/NS/Index.html

\section{NMR versus synchrotron radiation}

The completion of the world's largest nuclear magnetic resonance (NMR) facility at the campus of the RIKEN Genomic Sciences Center in Yokohama is an important step forward for Japan's structural genomics programme. NMR spectroscopy is a potential rival to synchrotron radiation in protein-structure analysis and, with RIKEN's new facility, Japanese scientists hope to play a key role in developing new instrumentation tools for structural genomics. Initially, the new facility will have 16 machines, and this number is expected to increase to 200 at its completion in 2002. Among others, the facility will host the world's most powerful NMR device.

Although some scientists are sceptical about whether NMR spectroscopy will ever replace crystallography as a research tool in structural biology, others argue that much could be done to improve NMR techniques, and that experimental performance could surge with the availability of new, more powerful instruments.

Yoshiyuki Yokoyama, who is responsible for RIKEN's structural genomics effort, believes that X-ray crystallography and NMR spectroscopy have their perspective advantages and that combining both techniques is likely to be the most beneficial strategy. "X-ray crystallography and NMR spectroscopy are complementary technologies," he says. 